\title{
TRIAGEM AUDITIVA NEONATAL UNIVERSAL: ÍNDICE DE EFETIVIDADE NO RETESTE DE NEONATOS DE UM HOSPITAL DA REDE PÚBLICA DE CAMPINAS
}

\author{
Universal neonatal screening: index of retest effectiveness \\ among newborns of a public hospital in Campinas - Brazil
}

\author{
Paloma Savioli Berni ${ }^{(1)}$, Elizabeth Oliveira Crepaldi de Almeida ${ }^{(2)}$, \\ Bárbara Carolina Teixeira Amado ${ }^{(3)}$, Nelson de Almeida Filho ${ }^{(4)}$
}

\section{RESUMO}

Objetivos: conhecer o índice de bebês avaliados na Triagem Auditiva Neonatal Universal de um hospital da rede pública de Campinas - São Paulo que concluíram o processo diagnóstico tendo falhado no teste de Emissões Otoacústicas Evocadas Transientes. Métodos: a amostra é composta por 1146 protocolos de bebês avaliados no Programa de Triagem Auditiva Neonatal Universal desse hospital, no período de julho de 2007 a abril de 2008. Resultados: a Triagem Auditiva Neonatal Universal mostrou efetividade de 69,4\%, porém, a partir do reteste dos bebês que falharam, o índice de sucesso no seguimento cai acentuadamente. Do total da amostra, 11 bebês apresentaram suspeita de perda auditiva, dos quais quatro apresentaram alteração. Conclusão: os dados sugerem a necessidade de aperfeiçoamento do programa.

DESCRITORES: Audiologia; Triagem Neonatal; Recém-Nascido; Diagnóstico Precoce; Otolaringologia

\section{INTRODUÇÃO}

A perda auditiva mesmo que discreta, pode alterar o desenvolvimento da comunicação oral ${ }^{1}$, e por

(1) Aluna do Curso de Graduação em Fonoaudiologia da Pontifícia Universidade Católica de Campinas; Bolsista de Iniciação Científica; Membro do Grupo de Estudo dos Aspectos Moleculares da Perda Auditiva da Universidade Estadual de Campinas, UNICAMP, Campinas, SP.

(2) Fonoaudióloga; Docente Titular do Curso de Fonoaudiologia da Pontifícia Universidade Católica de Campinas, PUCCAMP, Campinas, SP; Pesquisadora do Grupo de Estudo dos Aspectos Moleculares da Perda Auditiva da Universidade Estadual de Campinas, UNICAMP, Campinas, SP; Doutora em Educação.

(3) Aluna do Curso de Graduação em Fonoaudiologia da Pontifícia Universidade Católica de Campinas; Bolsista de Iniciação Científica; Membro do Grupo de Estudo dos Aspectos Moleculares da Perda Auditiva da Universidade Estadual de Campinas, UNICAMP, Campinas, SP.

(4) Aluno do Curso de Graduação em Medicina da Uiversidade de Taubaté; Membro do Grupo de Estudo dos Aspectos Moleculares da Perda Auditiva da Universidade Estadual de Campinas, UNICAMP, Campinas, SP.

Este trabalho faz parte da pesquisa: Avaliação do custo-benefício da triagem audiológica associada ao exame genético em neonatos que tem auxílio do CNPq - edital temático 026/2006.

Conflito de interesses: inexistente esta razão, foram implantados programas voltados à atenção neonatal para diagnóstico e acompanhamento audiológico, assim como de intervenção clínica-terapêutica ${ }^{2,3}$. A Triagem Auditiva Neonatal Universal (TANU) ${ }^{4}$ é o rastreamento auditivo em que todas as crianças devem ter acesso à triagem auditiva antes de um mês de vida, sendo que as crianças que falharem na triagem auditiva inicial e no reteste devem receber avaliação médica e audiológica adequada para confirmar a perda auditiva antes dos 3 meses de idade 5; crianças que apresentam indicadores de risco para perda auditiva devem ser acompanhadas a cada seis meses por três anos ${ }^{6}$.

A implementação da TANU por meio do teste das Emissões Otoacústicas Evocadas (EOAE) se baseia na energia acústica produzida na orelha interna que pode ser captada no conduto auditivo externo ${ }^{7}$, sendo que a resposta desaparece quando existir qualquer anomalia funcional na oreIha interna ${ }^{8}$. Esse teste foi escolhido por ser um procedimento que possibilita a testagem de grande número de recém-nascidos ${ }^{9}$, mas o ideal é ao identificar a criança de risco, encaminhar para confirmação da suspeita da deficiência auditiva e enquadrar 
em processos terapêuticos ${ }^{10}$, sendo de grande importância o trabalho multidisciplinar ${ }^{11}$.

Espera-se que aproximadamente 2 a $4 \%$ dos recém-nascidos em berçários de baixo risco e 5 a $7 \%$ em pacientes internados em unidades de terapia intensiva não passem no teste inicial ${ }^{12}$, sendo que o índice de falso positivo não deve ultrapassar $3 \%$ e o índice de falso negativo deve ser idealmente igual a zero ${ }^{3}$. As falhas no exame podem ocorrer devido ao barulho, descamações celulares presentes no conduto auditivo externo ou secreção na orelha média ${ }^{12}$, ou mesmo escolha equivocada de protocolo e da tecnologia para a população-alvo ${ }^{13}$. É importante que quando a triagem estiver alterada, os pais recebam informações sobre a importância da triagem para compreender os benefícios do diagnóstico precoce e intervenção ${ }^{14}$, além de maior adesão aos retornos ${ }^{9}$.

A prevalência da perda auditiva permanente pode aumentar até os 9 anos de idade, atingindo 205 por 100.000 pacientes ${ }^{15}$. Se esta afirmação é correta, sugere que a perda auditiva progressiva seja subestimada ${ }^{16}$, pois o primeiro diagnóstico tem sido realizado, em média, aos dois anos e meio de idade nos países desenvolvidos e, no Brasil, a idade média do diagnóstico varia em torno de três a quatro anos, podendo levar até dois anos para ser concluído ${ }^{17}$.

Um programa é considerado efetivo quando no mínimo 95\% das crianças que falharam na triagem auditiva inicial são acompanhadas ${ }^{3}$, com índice mínimo de retorno para seguimento de $70 \%{ }^{18} \mathrm{e} o$ reteste num prazo máximo de 30 dias de vida ${ }^{19}$. Em um estudo ${ }^{20}$, foram avaliados $99 \%$ do total de nascimentos, com índice de falha de $4,1 \%$, índice de falso-positivo de $3,6 \%$, média de idade no diagnóstico de 3,9 meses e a intervenção foi de 6,1 meses. A intervenção deve ocorrer o mais rápido possível, para buscar garantir desenvolvimento de fala e linguagem, o mais próximo do normal ${ }^{21}$.

É fundamental o conhecimento e a valorização dos profissionais de saúde no período pré e pósgestacional para que haja efetividade nesses programas ${ }^{22}$. Em um estudo referente à conduta dos pediatras ${ }^{23}$, encontrou-se que nos casos de alto risco, $92 \%$ disseram fazer encaminhamento ao otorrinolaringologista, $4 \%$ para triagem auditiva neonatal e avaliação audiológica, 4\% não especificaram.

Segundo o Joint Committee on Infant Hearing ${ }^{4}$ cerca de metade dos recém-nascidos que não passam na primeira triagem deixam de ter um apropriado seguimento para confirmar a presença de uma perda auditiva e/ou iniciar serviços adequados de intervenção precoce.

O presente estudo tem como objetivo geral conhecer o índice de bebês avaliados na TANU de um hospital da rede pública de Campinas - São Paulo que concluíram o processo diagnóstico tendo falhado no teste de EOAET.

\section{Objetivos específicos}

- Caracterizar a amostra de acordo com o período, idade e gênero da amostra;

- Calcular a porcentagem de bebês que passaram ou falharam no primeiro exame, no segundo exame e terceiro exame;

- Verificar em que momento ocorreu abandono, respeitando as etapas do protocolo utilizado o serviço;

- Verificar a porcentagem de exames concluídos e não concluídos.

\section{MÉTODOS}

Este estudo documental, autorizado pelo Diretor da Instituição, envolve uma amostra composta por 1146 protocolos de recém-nascidos avaliados no Programa de Triagem Auditiva Neonatal Universal de um hospital da rede pública situado na cidade de Campinas-SP, no período de julho de 2007 a abril de 2008. Esses protocolos contêm registro de dados de: identificação, antecedentes, indicadores de risco para surdez, resultados do teste de Emissões Otoacústicas Evocadas Transientes, registros de encaminhamentos consulta otorrinolaringológica, realização do exame Potencial Evocado Auditivo de Tronco Encefálico - PEATE, intervenção do Serviço Social; além de agendamento de retorno para monitoramento dos casos de risco ou dos que não compareceram ao atendimento na data marcada.

Os Protocolos obtidos no período deste estudo foram analisados com relação às informações sobre o seguimento de todo o processo diagnóstico dos bebês avaliados, que falharam na primeira avaliação. Esses dados foram utilizados para estabelecer o índice de participação dos bebês em todas as fases do processo de diagnóstico.

Segundo as informações da instituição, o exame de EOAET foi realizado com o equipamento ILO 292 OAE - Otodynamics, nas frequências de 1000 a $4000 \mathrm{~Hz}$, com intensidade de $70 \mathrm{~dB}$. O resultado foi registrado considerando as respostas das EOAET em relação sinal/ruído, tendo como critério: presente - respostas em três frequências ou mais e ausente - respostas em zero, uma ou duas frequências. Quando a avaliação no primeiro exame resultou em ausência de resposta, o recém-nascido foi encaminhado para segundo exame. Aqueles que falharam no segundo exame foram encaminhados para avaliação otorrinolaringológica e posterior terceiro exame se necessário. 
Este estudo foi submetido ao Comitê de Ética em Pesquisa da Pontifícia Universidade Católica de Campinas e autorizado pelo superintendente da Instituição, sob o protocolo de número 734/06.

Os dados foram analisados quantitativamente $e$ apresentados em figuras considerando-se a distribuição estatística percentual da amostra.

\section{RESULTADOS}

A idade dos recém-nascidos da amostra, que realizaram a TANU no período de julho de 2007 a abril de 2008, variou de 0 a 140 dias, com média de 7,8 dias. A maior parte foi avaliada com dois dias de vida $713(62,25 \%)$. A Figura 1 mostra a distribuição por sexo dos neonatos da amostra.

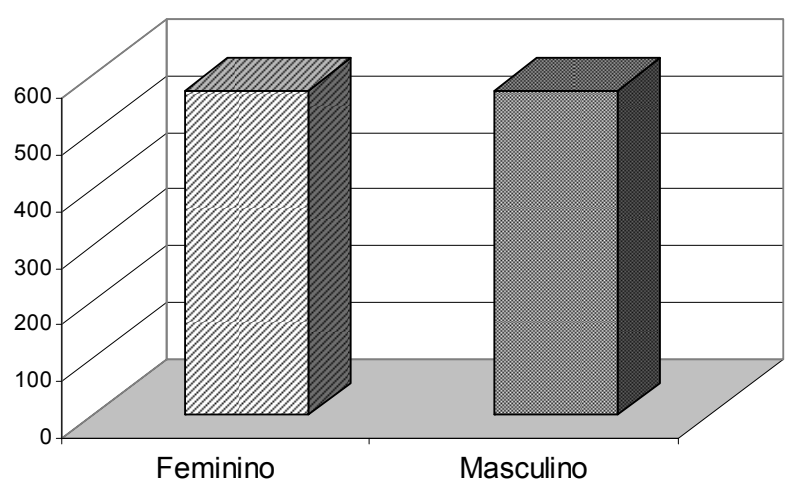

Figura 1 - Distribuição da amostra por gênero

A distribuição dos resultados dos exames de EOAET realizados na primeira avaliação encontrase na Figura 2.

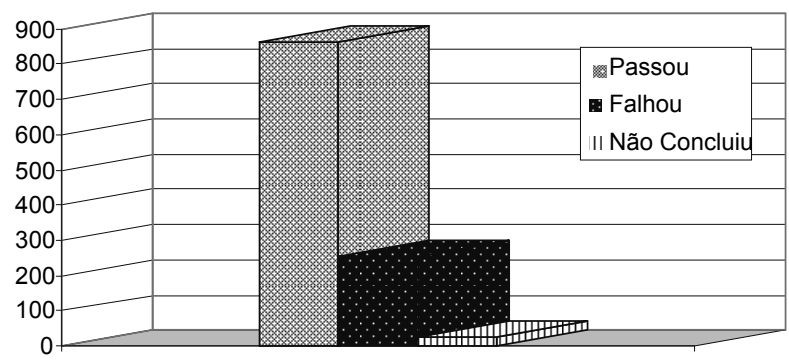

Figura 2 - Resultado das EOAET na TANU

Os que falharam ou não concluíram o primeiro exame foram encaminhados para reteste, totalizando 271 neonatos. A idade mínima no reteste foi de nove dias, a máxima de 113 dias, com média de 21,42 . A Figura 3 mostra os resultados do reteste.

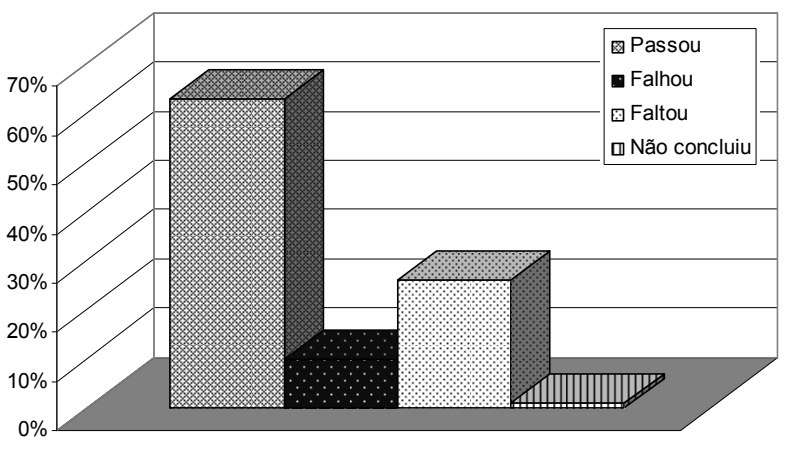

Figura 3 - Resultados do reteste com EOAET $(n=271)$

Os bebês que falharam no reteste foram encaminhados a uma terceira avaliação por meio das EOAET. A idade mínima observada entre os que compareceram foi de 23 dias, a máxima foi de 73 dias e a média foi 39,1 dias. Do total de 101 bebês a serem reavaliados, mais da metade (69) estava sem agendamento e 47 casos haviam sido encaminhados ao Serviço Social da instituição. A Figura 4 evidencia os resultados.

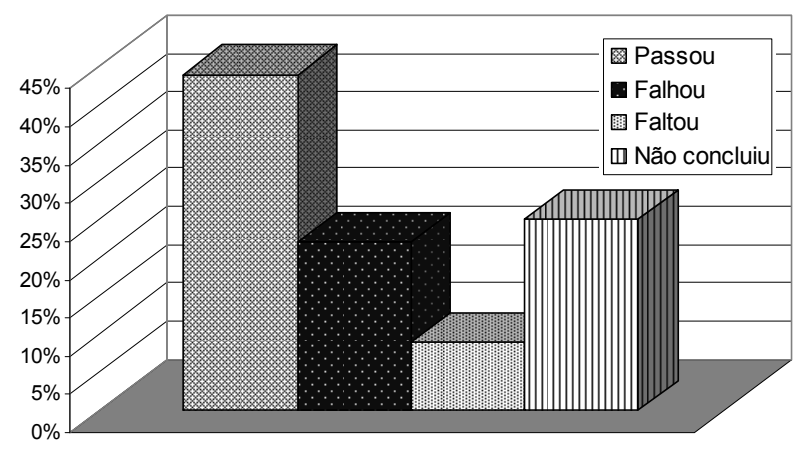

Figura 4 - Resultado da terceira avaliação por EOAET

Dos bebês encaminhados à terceira avaliação por meio das EOAET, 20 também foram encaminhados à consulta com otorrinolaringologista, dos quais 11 foram encaminhados para realização do PEATE. Destes, quatro faltaram, sendo dois por estarem doentes; um não precisou fazer esse exame (o paciente realizou EOA anteriormente ao PEATE com resultado presente); dois tiveram resultado normal e quatro apresentaram alteração. A Figura 5 sintetiza as ocorrências registradas na amostra, quanto aos resultados dos exames, encaminhamentos e faltas. 


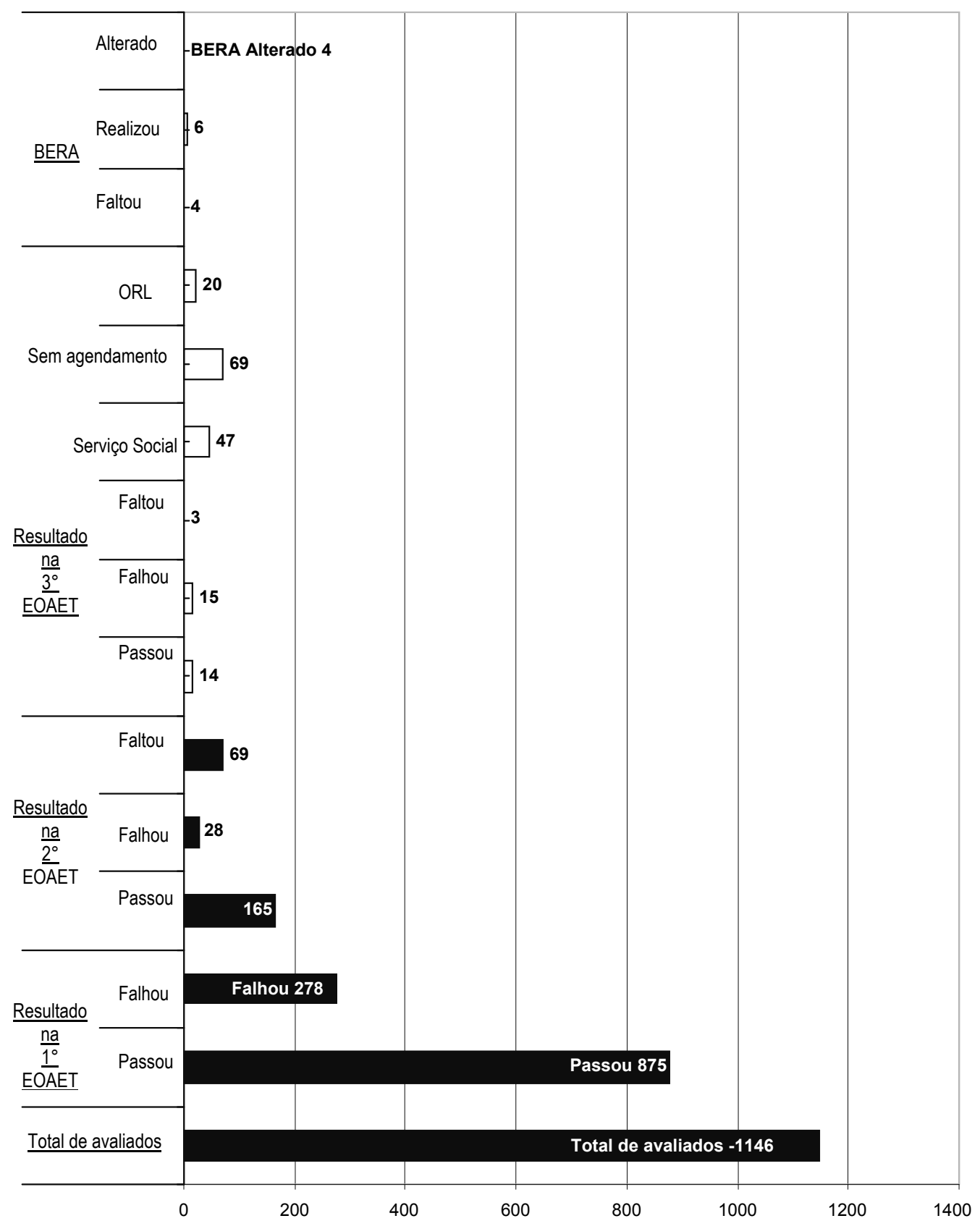

Figura 5 - Resultado do processo de Triagem Auditiva Neonatal Universal e seguimento

\section{DISCUSSÃO}

Todos os recém-nascidos, ou seja, $100 \%$ das crianças foram testadas na TANU de acordo com o número de nascidos vivos do hospital.

O protocolo adotado na maternidade em que o estudo foi realizado ocorre da seguinte forma: os bebês que falham no reteste são encaminhados para o otorrinolaringologista e, após esse acompanhamento, são encaminhados para realizar uma nova EOAET. Se nessa terceira EOAET, o bebê ainda falhar será encaminhado para a realização do
PEATE e, caso o bebê apresente alteração nesse processo, terá acompanhamento para estimulação auditiva.

A idade dos neonatos, que realizaram a TANU no período de julho de 2007 a abril de 2008 , variou de 0 a 140 dias, com média de 7,8 dias, números compatíveis com o Joint Committee on Infant Hearing ${ }^{4}$, que preconiza a triagem dos neonatos no primeiro mês de vida.

Os dados colhidos evidenciam que, de uma amostra de 1146 neonatos, 271 falharam nas EOAET. No reteste, 69 bebês faltaram e não tiveram 
um segundo reteste agendado, e destes 47 foram encaminhados ao Serviço Social. Assim, o índice de efetividade no reteste foi de $69,4 \%$. Organismos internacionais e nacionais estabelecem como bem sucedido um programa de triagem auditiva neonatal no qual $95 \%$ dos neonatos que falharam na triagem auditiva inicial recebem acompanhamento ${ }^{4}$.

Os casos foram encaminhados ao Serviço Social para orientar as famílias a darem seguimento ao processo de avaliação. Mas, do total dos bebês indicados ao terceiro exame, 69 não apresentavam registro de data marcada para sua realização. Assim, mais da metade dos casos deixaram de ser avaliados em um prazo compatível com o preconizado pelo $\mathrm{JCIH}^{4}$ e pela literatura ${ }^{3,18,19}$.

$\mathrm{Na}$ amostra estudada, dos 101 bebês que falharam ou faltaram à segunda avaliação, 32 compareceram ao terceiro agendamento do teste de EOAET. Isso representa $31,68 \%$, taxa bem abaixo de $95 \%$ de avaliações para se considerar o programa bem sucedido pelo Comitê Brasileiro Sobre Perdas Auditivas na Infância - $1999^{3}$ e pelo Joint Committee on Infant Hearing Year ${ }^{4}$.

Adicionalmente, dos bebês indicados à terceira avaliação, alguns são encaminhados à consulta com otorrinolaringologista em função da presença de indicadores ao exame com otoscópio realizado durante o processo de avaliação fonoaudiológica ou presença de outros problemas, como ronco, chiado, entre outros registrados no protocolo do exame.
Os dados evidenciam que a falha no acompanhamento ocorreu no segundo passo do Programa. Mesmo com o agendamento de reteste já na maternidade, registra-se um índice considerável de ausência, já que 284 (24,8\%) dos bebês agendados faltaram e não tiveram outra avaliação agendada, o que somado às faltas na terceira EOAET e no PEATE eleva para $312(27,3 \%)$ o índice de perdas. No entanto, o índice de exames não concluídos foi de apenas $n=68$ (6\%) e o índice de concluídos foi de 1077 (94\%), o que indica a efetividade do programa.

\section{CONCLUSÃO}

Os dados analisados sugerem a necessidade de reavaliação e estabelecimento de metas compatíveis e de contra-medidas para evitar falhas no agendamento e faltas por parte dos casos agendados. A intervenção da Assistente Social não se mostrou suficiente para o comparecimento da mãe. Dessa forma, sugere-se contato direto com os agentes de saúde de referência da família.

\section{AGRADECIMENTOS}

À Fonoaudióloga Natália Gallate Jorge e Lívia Viti. À FAPESP (Bolsa de Iniciação Científica).

Ao CNPq pelo auxílio concedido para o desenvolvimento da pesquisa.

\section{ABSTRACT}

Purpose: to know the index of babies who failed in the EOAET diagnosis process in the Universal Neonatal Auditory Scan Program of an public hospital in Campinas, São Paulo, Brazil. Methods: the sample is composed of 1146 protocols of babies evaluated in the Universal Neonatal Auditory Scan Program in this hospital, in the period of July 2007 to April 2008. Results: Universal Neonatal Auditory Scan presented effectiveness of $69.4 \%$, although the retest of the babies that failed in the EOAET diagnosis have shown that the success in the follow up process goes down significantly. From the total of the sample, 11 babies were suspected to have auditory loss and four had some alteration. Conclusion: data suggesting that the program needs to be improved.

KEYWORDS: Audiology; Neonatal Screening; Infant, Newborn; Early Diagnosis; Otolaryngology

\section{REFERÊNCIAS}

1. Borges CAB, Moreira LMO, Pena GM, Fernandes $\mathrm{FR}$, Borges BCB, Otani BH. Triagem auditiva neonatal universal. Rev Arq Int Otorrinolaringol. 2006; 10(1):28-34.
2. Azevedo MF. Desenvolvimento auditivo de crianças normais e de alto-risco. [tese] São Paulo (SP): Universidade Federal de São Paulo; 1993. $329 \mathrm{f}$.

3. Comitê Brasileiro sobre Perdas Auditivas na Infância. 1999. Disponível em: URL: http://www. 
unifesp.br/dotorrino/orl/graduacao/roteiros/surdez inf.doc. Acesso em: 17 set. 2007.

4. Joint Comittee on Infant Hearing. Year 2007. Position Statement: Principles and Guidelines for Early Hearing Detection and Intervention Programs. Pediatrics. 2007; 120:798-817.

5. Araújo FCRS, Almeida EC, Arroyo NL. Avaliação audiológica do recém-nascido. In: Almeida EC, Modes LC. Leitura de prontuário-avaliação e conduta fonoaudiológica com o recém-nato de risco. Rio de Janeiro: Revinter, 2005. p 61-80.

6. Manfredi AFS, Santos CB. Intervenção fonoaudiológica junto a mães ou responsáveis por recém-nascidos de risco para deficiência auditiva, precocemente detectada. Medicina. 2002; 35(1): 70-7.

7. Tochetto TM, Gatto Cl. Deficiência auditiva infantil: implicações e soluções. Rev CEFAC. 2007; 9(1):110-5.

8. Uchôa NT, Procianoy RS, Lavinsky L, Sleifer P. Prevalência de perda auditiva em recém-nascidos de muito baixo peso. J Pediatr. 2003; 79(2):123-8.

9. Durante AS, Carvallo RMM, Costa MTZ, Cianciarullo MA, Voegels RL, Takahashi GM, et al. Programa de triagem auditiva neonatal: modelo de implementação. Rev Arq Int Otorrinolaringol. 2004; 8(1):56-62.

10. Weiss KM. Pediatras e neonatologistas: detecção precoce da deficiência auditiva. [monografia] Porto Alegre (RS): CEFAC - Saúde e Educação; 1999.

11. Pádua FGM, Marone S, Bento RF, Carvallo RMM, Durante AS, Soares JC, et al. Triagem auditiva neonatal: um desafio para sua implantação. Rev Arq Int Otorrinolaringol. 2005; 9(3):189-4.

12. Widen JE, Bull RW, Folson RC. Newborn hearing screening: what it means for providers of early intervention services. Infants Young Child. 2003; 16(3):249-57.

13. Barreira-Nielsen C, Futuro Neto HA, Gattaz G. Processo de implantação de programa de saúde auditiva em duas maternidades públicas. Rev Soc Bras Fonoaudiol. 2007; 12(2):99-105.
14. Arnold CL, Davis TC, Humiston SG, Bocchini JA, Bass PT, Bocchini A, et al. Infant hearing screening: stakeholder recommendations for parent-centered communication. Pediatrics. 2006; 117(5Pt2): 341-54.

15. Fortunun HM, Summerfield $Q$, Marshall $D H$, Davis AC, Bamford JM. Prevalence of permanent childhood hearing impairment in the United Kingdom and implications for universal neonatal screening: questionnaire based ascertainment study. BMJ. 2001; 323:536.

16. Kennedy C, McCann D. Universal neonatal hearing screening moving from evidence to practice. Arch Dis Child Fetal Neonatal. 2004; 89(5):F378-F83.

17. Marone MR, Lichtig I, Marone SAM. Recémnascidos gerados por mães com alto risco gestacional: estudo das emissões otoacústicas produtos de distorção e do comportamento auditivo. Rev Bras Otorrinolaringol. 2002; 68(2):230-7.

18. Comitê Brasileiro sobre Perdas Auditivas na Infância. Período neonatal. J Pediatria. 2001; 77:1. 19. Marques TR, Mendes PC, Bochnia CFP, Jacob LCB, Roggia SM, Marques JM. Triagem auditiva neonatal: relação entre banho e índice de reteste. Rev Bras. Otorrinolaringologia. 2008; 74(3):375-81. 20. Connolly JL, Carron JD, Roark SD. Universal newborn hearing screening: are we achieving the Joint Committee on Infant Hearing $(\mathrm{JClH})$ objectives? Laryngoscope. 2005; 115(2):232-6.

21. Ribeiro FG, Mitre El. Avaliação do conhecimento sobre triagem auditiva neonatal de pacientes no pós-parto imediato. Rev CEFAC. 2004; 6(3):294-9. 22. Hilú MRPB, Zeigelboim BS. O conhecimento, a valorização da triagem auditiva neonatal e a intervenção precoce da perda auditiva. Rev CEFAC. 2007; 9(4):563-70.

23. Zocoli AMF, Riechel FC, Zeigelboim BS, Marques JM. Audição: abordagem do pediatra acerca dessa temática. Rev Bras Otorrinolaringol. 2006; 72(5):617-23.

DOI: 10.1590/S1516-18462009005000034

RECEBIDO EM: 02/06/2008

ACEITO EM: 16/03/2009

Endereço para correspondência:

Nelson de Almeida Filho

Av. Maria Martins Otobonni, 210

Jacareí - SP

CEP: 12306-700

E-mail: nelson_almeida@uol.com.br 